\title{
Improving Touschek lifetime in ultralow-emittance lattices through systematic application of successive closed vertical dispersion bumps
}

\author{
J. Breunlin, 'S. C. Leemann, and $\AA$. Andersson \\ MAX IV Laboratory, Lund University, SE-22100 Lund, Sweden
}

(Received 4 December 2015; published 9 June 2016)

\begin{abstract}
In present ultralow-emittance storage ring designs the emittance coupling required for the production of vertically diffraction-limited synchrotron radiation in the hard $\mathrm{x}$-ray regime is achieved and in many cases surpassed by a correction of the orbit and the linear optics alone. However, operating with a vertical emittance lower than required is disadvantageous, since it decreases Touschek lifetime and reduces brightness due to the transverse emittance increase from intrabeam scattering. In this paper we present a scheme consisting of closed vertical dispersion bumps successively excited in each arc of the storage ring by skew quadrupoles that couple horizontal dispersion into the vertical plane to a desired level and thereby raise the vertical emittance in a controlled fashion. A systematic approach to vertical dispersion bumps has been developed that suppresses dispersion and betatron coupling in the straight sections in order to maintain a small projected emittance for insertion devices. In this way, beam lifetime can be significantly increased without negatively impacting insertion device source properties and hence brightness. Using simulation results for the MAX IV $3 \mathrm{GeV}$ storage ring including magnet and alignment imperfections we demonstrate that Touschek lifetime can be increased by more than a factor 2 by adjusting the vertical emittance from $1.3 \mathrm{pm}$ rad (after orbit correction) to $8 \mathrm{pm}$ rad (after application of dispersion bumps) using two to three independent skew quadrupole families all the while ensuring deviations from design optics are restrained to a minimum.
\end{abstract}

DOI: 10.1103/PhysRevAccelBeams.19.060701

\section{INTRODUCTION}

Today's ultralow-emittance (ULE) storage rings are based on multibend achromat (MBA) lattices. In such storage rings the emittance coupling required to operate at the diffraction limit in the vertical plane is comparably high. With orbit corrections and linear optics corrections applied the vertical emittance resulting from imperfections (magnet and alignment errors) can, however, become extremely low, lower in fact than required to be diffractionlimited at the wavelengths of interest. This is a highly undesirable situation because of the resulting Touschek lifetime penalty [1-3] as well as brightness limitations caused by the increased emittance blowup from intrabeam scattering (IBS) [4].

We propose therefore to drive successive closed vertical dispersion bumps (SCVDBs) in the MBA arcs around the storage ring. Ideally, the vertical emittance is then created directly via quantum excitation in the bending magnets. Closing the vertical dispersion bump at the end of the arc ensures that the straights remain dispersion free. In this way, and by ensuring low betatron coupling in the straights, the

\footnotetext{
*jonas.breunlin@maxiv.lu.se

Published by the American Physical Society under the terms of the Creative Commons Attribution 3.0 License. Further distribution of this work must maintain attribution to the author $(s)$ and the published article's title, journal citation, and DOI.
}

electron beam in the insertion devices (IDs) provides small source properties in order to produce diffraction-limited synchrotron radiation (SR) and maintain high levels of transverse coherence [5]. The Touschek lifetime is, however, substantially improved by the increase in vertical emittance since it can be shown to scale like $\tau \propto \sqrt{\mathcal{E}_{\text {II }}}$ (see Sec. II A).

As will be shown in this paper, a SCVDB lattice with properly closed vertical dispersion bumps but otherwise only minor deviations from the design lattice can be found. In such a lattice the dynamic aperture (DA) remains high and there is only negligible betatron coupling in the IDs where the vertical acceptance limitations are located. Therefore, lifetime can be considerably improved while injection efficiency remains excellent.

Vertical dispersion bumps have been proposed before. In one set of applications a local closed bump is required to achieve a transverse separation within an energy-modulated beam [6]; contrary to what is proposed in this paper such a bump is created explicitly within the ID. In other applications vertical dispersion waves for lifetime improvement have been proposed $[7,8]$, however, in such cases the vertical dispersion follows a wave extending throughout both straights and arcs. It therefore improves the Touschek lifetime, but it also removes achromaticity and alters the source properties in the IDs. In ULE storage rings this is no longer an acceptable perturbation.

In contrast to such attempts, the method proposed here takes a more fundamental approach where achromaticity 
and beam properties in the IDs are introduced as fixed boundary constraints. The vertical dispersion is then tailored in specific locations away from the IDs to exactly match lifetime requirements while respecting all boundary constraints. This systematic approach of successive closed vertical dispersion bumps and three examples of applications to the MAX IV $3 \mathrm{GeV}$ storage ring along with estimates of the achievable performance, based on results from 6D tracking with Tracy-3 [9], will be presented in this paper.

\section{BACKGROUND AND PRINCIPLE}

\section{A. Touschek lifetime and vertical emittance}

Touschek scattering is the dominant beam loss mechanism in present storage ring light sources. The effect of vertical emittance on Touschek lifetime can be derived from [1-3]

$$
\frac{1}{\tau}=\frac{r_{e}^{2} c q}{8 \pi e \gamma^{3} \sigma_{s} C} \cdot \oint_{C} \frac{F\left(\left[\frac{\delta_{\mathrm{acc}}(s)}{\gamma \sigma_{x^{\prime}}(s)}\right]^{2}\right)}{\sigma_{x}(s) \sigma_{y}(s) \sigma_{x^{\prime}}(s) \delta_{\mathrm{acc}}^{2}(s)} d s,
$$

where $r_{e}$ denotes the classical electron radius, $c$ the vacuum velocity of light, $e$ the elementary charge, $C$ the circumference of the storage ring, $q$ the bunch charge and $\gamma$ the Lorentz factor of the beam. The horizontal and vertical rms beam sizes are $\sigma_{x}$ and $\sigma_{y}$, respectively, while $\sigma_{s}$ denotes the rms bunch length, $\delta_{\text {acc }}(s)$ the local momentum acceptance and $\sigma_{x^{\prime}}(s)$ the horizontal rms beam divergence for an electron at $x \approx 0$, where Touschek scattering events are expected to occur. Finally, the function $F(x)$ is defined as

$$
F(x)=\int_{0}^{1}\left[\frac{1}{u}-\frac{1}{2} \ln \left(\frac{1}{u}\right)-1\right] \exp \left(-\frac{x}{u}\right) d u .
$$

Under the assumption that both vertical dispersion and betatron coupling are negligible contributions to the vertical beam size $\left(\sigma_{y}(s) \approx \sqrt{\mathcal{E}_{\mathrm{II}} \beta_{\mathrm{II}}(s)}\right)$ the Touschek lifetime then scales like

$$
\tau \propto \sqrt{\mathcal{E}_{\mathrm{II}}}
$$

Hence a substantial improvement of the Touschek lifetime can be achieved by increasing the vertical emittance.

\section{B. ULE lattices and vertical dispersion}

A typical example for an ULE storage ring lattice is the design lattice of the MAX IV $3 \mathrm{GeV}$ storage ring [10]. It shows a low horizontal dispersion in the arcs while the long straights are dispersion-free (achromatic lattice), see Fig. 1. Since the vertical dispersion is zero by design (flat lattice), the only sources of vertical emittance in the real storage ring, apart from quantum excitation from SR emission (amounting to less than $0.1 \mathrm{pm} \mathrm{rad}$ ), are betatron coupling and spurious vertical dispersion, resulting from imperfections such as magnet and alignment errors.

It is common practice to minimize both vertical dispersion and betatron coupling in order to improve the $\mathrm{DA}^{1}$ and to reduce beam losses on narrow vertical acceptances (usually found in the long straights as a consequence of narrow-gap chambers and in-vacuum IDs). This can be achieved following the widely used LOCO [14] approach: The betatron coupling is determined from off-diagonal elements in the orbit response matrix whereas vertical orbits from $\mathrm{rf}$ frequency shifts yield the vertical dispersion response matrix. Betatron coupling and vertical dispersion are then minimized by inversion of sensitivity matrices from a calibrated machine model, which describes the influence of correcting skew quadrupoles on the orbit and dispersion response matrix, for an example see [15]. However, the resulting low emittance coupling might reduce the Touschek lifetime to unacceptable values. Negative consequences of a short beam lifetime are an increased top-up rate and possibly radiation issues. Furthermore, a low emittance coupling further increases IBS blowup which is already severe in ULE lattices, when storing high current at medium energy [4].

To alleviate this problem the vertical emittance might be created from vertical dispersion in the arcs only up to the maximum permissible level (diffraction limit). In the presence of vertical dispersion the natural emittance for the vertical plane yields $[16,17]$ :

$$
\mathcal{E}_{\mathrm{II}} \approx C_{q} \gamma^{2} \frac{I_{5, \mathrm{II}}}{I_{2}} \quad \text { with } \quad I_{5, \mathrm{II}}=\oint \frac{\mathcal{H}_{\mathrm{II}}}{|\rho|^{3}} d s
$$

where the constant is $C_{q} \approx 3.823 \times 10^{-13} \mathrm{~m}, I_{2}$ and $I_{5, \text { II }}$ are the second and fifth (vertical) synchrotron radiation integrals and the bending radius is $\rho\left(\rho=\rho_{x}\right.$ in a flat machine). The chromatic invariant in the vertical plane is defined as

$$
\mathcal{H}_{\mathrm{II}}(s)=\gamma_{\mathrm{II}} \eta_{\mathrm{II}}^{2}+2 \alpha_{\mathrm{II}} \eta_{\mathrm{II}} \eta_{\mathrm{II}}^{\prime}+\beta_{\mathrm{II}} \eta_{\mathrm{II}}^{\prime 2}
$$

By excitation of skew quadrupole magnets horizontal dispersion can be coupled into the vertical plane, which, together with the excited betatron coupling $[18,19]$ increases the vertical emittance [20].

With a suitable set of skew quadrupoles a local vertical dispersion bump with locally increased betatron coupling can be created. Once a dispersion bump has been designed for one achromat, the principle is leveraged by systematically applying it to each achromat, resulting in SCVDBs around the entire ring. Opening and closing each dispersion bump within the same arc ensures that the straight sections remain dispersion-free. If in addition to closing the vertical

\footnotetext{
${ }^{1}$ Maintaining sufficient DA is a crucial requirement considering off-axis injection e.g. [11], which is being employed in several new ULE rings $[10,12,13]$.
} 


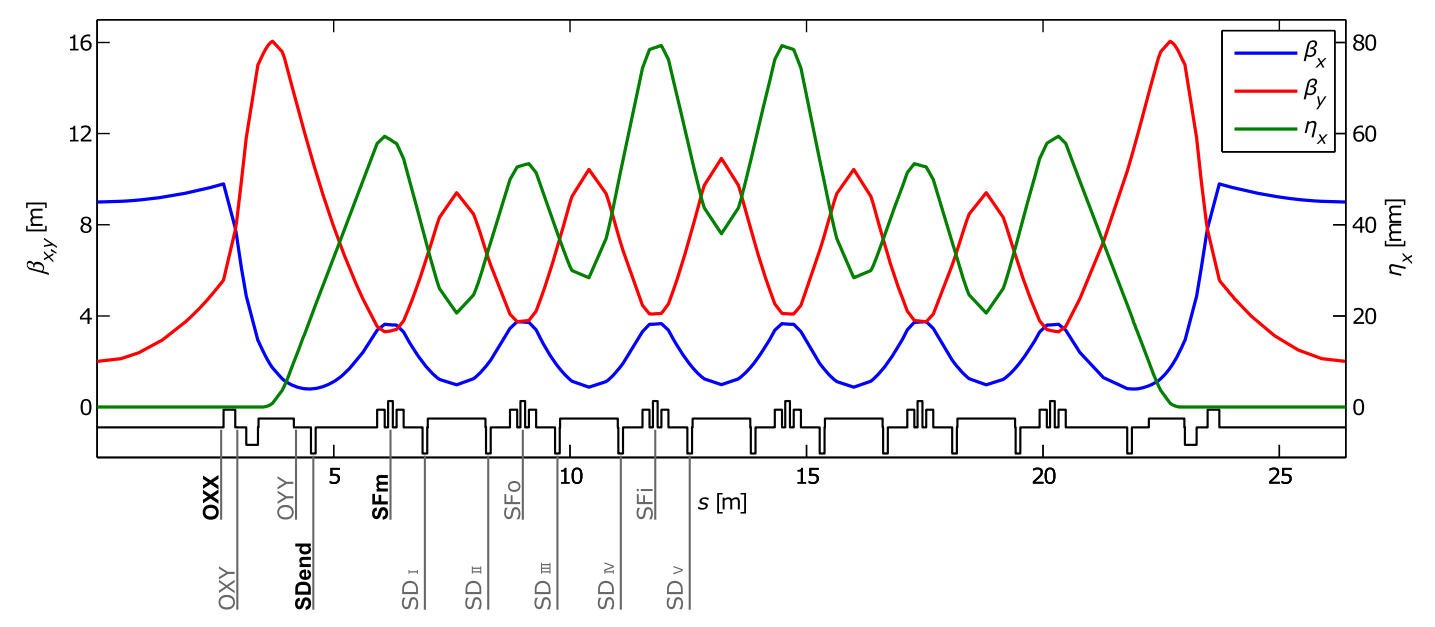

FIG. 1. Design optics of the MAX IV $3 \mathrm{GeV}$ storage ring in one achromat. The design vertical dispersion is zero everywhere. The positions of the magnets are indicated at the bottom in black. The positions and names of sextupole and octupole magnets, equipped with auxiliary coils to generate skew quadrupole gradients, are indicated below. The sequence of magnets repeats mirror-symmetrically throughout the second half of the achromat.

dispersion bumps the betatron coupling is restricted to the arcs the increase in beam size is also mainly restricted to locations in the arcs. The source properties of IDs in the long straights ${ }^{2}$ on the other hand are not altered, apart from the increase in vertical emittance, which is, however, adjusted below the diffraction limit.

In the simplest case and if no assumptions on the phase advance between skew quadrupoles are made, a closed vertical dispersion bump can be created with three skew quadrupoles. However, the dispersion function in the horizontal plane and beta functions present additional constraints. Since every deviation from the design lattice presents a potential source of undesired effects such as betatron coupling in the long straights or nonlinear optics detuning, the implementation of the vertical dispersion bumps must be done under consideration of those lattice functions. A certain flexibility in the choice of skew quadrupoles is helpful. It is therefore of great advantage that a large number of potential skew quadrupoles along the arcs have been built into the MAX IV $3 \mathrm{GeV}$ storage ring from the start (Fig. 1). The task remaining is to identify those skew quadrupoles and their gradients that create the vertical dispersion as desired in an efficient way and with a minimum deviation from the design optics.

\section{Vertical dispersion bump design using a SVD-based algorithm}

Closed expressions for the electron optics, such as beta functions and dispersion, as a function of the gradients of a set of skew quadrupoles can in principle be derived within the framework of linear approximation (e.g. [21]). Finding suitable dispersion distributions as analytical solutions of

\footnotetext{
${ }^{2}$ The MAX IV $3 \mathrm{GeV}$ storage ring design does not include bending magnet SR source points.
}

such a system of equations, however, becomes impractical for large numbers of skew quadrupole magnets. Instead, our systematic approach to SCVDBs is based on the recording of the lattice response to skew quadrupole magnet excitations, in order to generate a sensitivity matrix. Inverting this matrix a skew quadrupole setting can then be found that generates a dispersion bump with the desired properties. The required optics calculations are carried out with e.g. Tracy-3 and are applied to the design magnetic lattice of the storage ring.

The implementation of the dispersion bump as well as undesired deviations from the design lattice can be characterized by a set of key parameters e.g. the vertical dispersion in the bending magnets (where vertical emittance is created) as well as the beta functions and dispersion in SR source points or in regions of narrow apertures. The response of the lattice, given by all key parameters $\vec{p}=p_{1} \ldots p_{n}$, is then recorded as a function of the gradients of a set of skew quadrupoles $\vec{k}=k_{1} \ldots k_{m}$. This skew quadrupole setting may include many potentially available skew quadrupoles, since the selection of the most efficient skew quadrupoles follows in a later step. Systematic variations in skew quadrupole strengths $\vec{k}$ and recordings of the related responses $\vec{p}$ subsequently form the matrices $\mathcal{P}_{j, n}$ and $\mathcal{K}_{j, m}$, where $j$ denotes the index of the generated set of skew quadrupole setting and lattice response.

With the pseudoinverse of the $\mathcal{P}_{j, n}$ matrix, calculated via SVD (singular value decomposition) [22], the sensitivity matrix $\mathcal{M}$ is calculated as

$$
\mathcal{M}_{n, m}=\mathcal{P}_{n, j}^{-1} \cdot \mathcal{K}_{j, m},
$$

with which the skew quadrupole strengths $\vec{k}_{\mathrm{t}}$, required to approach a defined target $\vec{p}_{\mathrm{t}}$ in the key parameter space, can be determined as follows: 


$$
\vec{k}_{\mathrm{t}}=\vec{p}_{\mathrm{t}} \cdot \mathcal{M}
$$

This target $\vec{p}_{\mathrm{t}}$, expressible in terms of the key parameters, may represent the design lattice including the desired dispersion bump, or may be a more relaxed but still tolerable deviation from it. Once the lattice is adjusted for the setting $\vec{k}_{\mathrm{t}}$ and the lattice response is recorded, the success in approaching the target parameters is evaluated. In an iterative process the parameter space, given by the combinations and gradients of the available skew quadrupoles, is searched for suitable dispersion bump implementations.

In addition to the direct evaluation of the implemented dispersion bumps, the analysis of the sensitivity matrix itself provides valuable information. Due to lattice symmetry several groups of potential skew quadrupoles might be located where the ratios of the betatron functions and the horizontal dispersion are similar, and are therefore expected to have similar effects on the optics. Such a linear dependence presents an inefficient use of skew quadrupoles and should be avoided. For testing this the sensitivity matrix is written in its factorized form as follows (SVD):

$$
\mathcal{M}=U \Sigma V^{T}
$$

Here $U$ and $V$ are orthogonal matrices and $\Sigma$ is a $n \times m$ matrix with the singular values of $\mathcal{M}$ along its diagonal. Any inefficiently implemented dispersion bump can then be identified by a smaller number of nonvanishing singular values (the rank of the sensitivity matrix $\mathcal{M}$ ) compared to the number of employed skew quadrupoles. Furthermore, the sensitivity of each of the individual skew quadrupoles to the key parameters can be studied. This offers a systematic way to evaluate, compare, and improve dispersion bump implementations and their effect on the optics.

In practice the target parameters can often not be simultaneously fulfilled, but only approached. This can be caused by a dependency of the skew quadrupoles or just because the number of available skew quadrupole families is lower than the number of target parameters $(m<n)$, which leads to an underdetermined system of equations. Under these conditions trade-offs between the boundary conditions become necessary and can be investigated by variations of the target parameters within tolerable limits. An example for this is discussed in Sec. III A.

Applying this systematic approach allows finding a set of suitable skew quadrupoles and their individual excitation required to increase the Touschek lifetime efficiently by an increase in vertical emittance, while respecting the defined boundary conditions. An added benefit of this approach compared to a derivation from closed expressions is generality since no solutions have to be a priori excluded. Furthermore, the method allows quantifying the cost in terms of deviation from the design optics, possibly connected to an increase in beam size in IDs, and the achieved lifetime gain as a function of effort. Verification of the implemented SCVDBs can be performed by measuring a vertical dispersion response matrix and applying a LOCO fit to the lattice. With a suitable emittance monitor, as it is in preparation for the MAX IV $3 \mathrm{GeV}$ storage ring [23], the vertical emittance can be measured in a nondestructive way. This allows the controlled implementation and verification of SCVDBs even during user shifts.

\section{CASES AND RESULTS}

We use the MAX IV $3 \mathrm{GeV}$ storage ring to showcase our approach. Throughout the design phase of this storage ring it has been assumed that the vertical emittance would be adjusted to desired values. In the Detailed Design Report [23] the vertical emittance was specified to be $8 \mathrm{pm}$ rad, aiming for diffraction limited operation with $1 \AA$ SR. Later optimizations, aiming for maximum brightness and transverse coherence, called for $2 \mathrm{pm}$ rad vertical emittance [5].

Examples for how SCVDBs can be implemented in a real machine in order to meet such emittance requirements will now for the first time be demonstrated in detail. We present three design cases of SCVDB lattices to demonstrate how, with varying effort, the boundary constraints and target parameters can be enforced to required levels. Apart from the varying effort, represented by the number of individually powered skew quadrupole families (number of magnet power supplies), the cases are designed under slightly different trade-offs of the boundary constraints, to achieve the goal of a scalable Touschek lifetime increase with the least effect on the design optics.

\section{A. Boundary constraints and target parameters}

The MAX IV $3 \mathrm{GeV}$ storage ring provides 12 potential skew quadrupole families. This high number is achieved by auxiliary coils available on all sextupole and octupole magnets that can be powered (among others) as skew quadrupoles. Each of these magnets has an effective length of $0.10 \mathrm{~m}$ and a maximum normalized skew quadrupole gradient of $0.10 \mathrm{~m}^{-2}$ to $0.26 \mathrm{~m}^{-2}$, depending on the exact magnet type. The positions of all available auxiliary coils on sextupole and octupole magnets in the lattice are indicated in Fig. 1 for one half of the achromat. In accordance with the lattice symmetry the skew quadrupoles on mirror-symmetric positions within the achromat are paired and kept at identical gradients for the cases presented in this study. The presented cases presume that these skew quadrupole pairs are powered in each of the 20 achromats to generate identical vertical dispersion bumps around the entire storage ring. Therefore, one independent family of skew quadrupoles consists of 40 equally powered skew quadrupoles, and the lattice symmetry is unbroken (excluding machine imperfections).

Four of the five target parameters are set in order to minimize deviations from the design optics: Two 
parameters, $\beta_{x}=9.0 \mathrm{~m}$ and $\beta_{y}=2.0 \mathrm{~m}$ in the center of the long straight, prevent excessive growth of the beta functions compared to the design optics. The main purpose of this target is to avoid an increase in beam size in the ID. Stipulating this target also prevents large betatron coupling in the long straights as an indirect effect. In the ideal design lattice the horizontal and vertical dispersion $\eta_{x}$ and $\eta_{y}$ are zero in the long straights to ensure a sufficiently small source size in the IDs. In order to maintain this achromaticity, two additional targets are stipulated: $\left|\eta_{x}\right|<6 \mathrm{~mm}$ and $\left|\eta_{y}\right|<0.5 \mathrm{~mm}$ in the long straights. This ensures that dispersive contributions to the beam size remain negligible in the IDs for the assumed energy spread of $\sigma_{\delta}=0.8 \times 10^{-3}$. Since there are no bending magnet SR sources in the MAX IV $3 \mathrm{GeV}$ storage ring no constraints are made to maintain source properties in the arcs. In principle this could however be done in the same manner as in the long straights. The fifth target parameter is related to the amplitude of the vertical dispersion bump. For this purpose one key parameter is introduced that depends on the vertical dispersion in the bending magnets, since this is where vertical emittance is generated. The exact amplitude of the vertical dispersion bump is, however, not controlled by a target parameter. This problem is addressed in Sec. III C. Solutions where the vertical dispersion bump is opened as far upstream as possible in the arc (and closed as far downstream as possible) are preferred since they allow for lower vertical dispersion (more bends are involved in increasing the vertical emittance). In the cases presented here, this is achieved by using skew quadrupoles close to the long straights, rather than those around the center of the arc.

\section{B. Motivation of cases}

In the following a brief motivation for the design of the three presented cases is given. A detailed and comparative

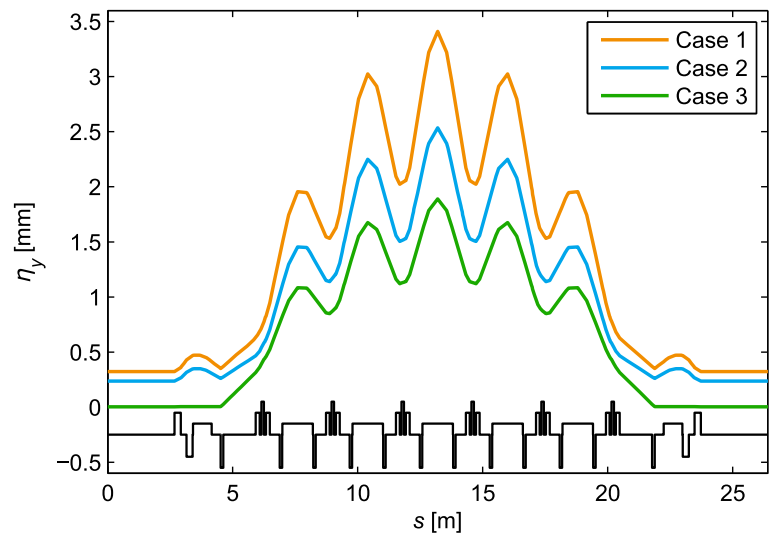

FIG. 2. Ideal vertical dispersion in Cases 1 to 3. Approximately $6.6 \mathrm{pm}$ rad of vertical emittance is created in the ideal lattice which gives on average $7.9 \mathrm{pm}$ rad when including imperfections. investigation can be found in the following sections. The vertical dispersion created in one achromat is shown in Fig. 2 for the three SCVDB cases. The vertical emittance created by these three cases in the ideal lattice (i.e. without imperfections) is $6.6 \mathrm{pm} \mathrm{rad}$.

Case 1 demonstrates that a considerable gain in Touschek lifetime can be achieved using just a single skew quadrupole family. In this case the vertical dispersion bump

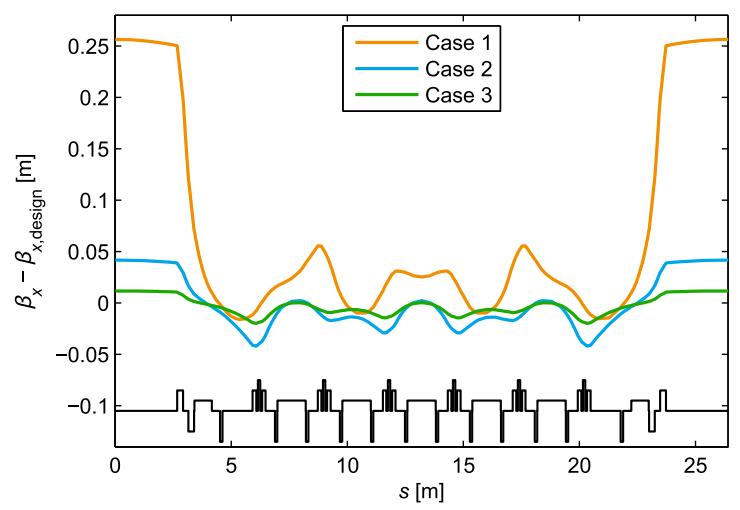

(a) Horizontal beta functions

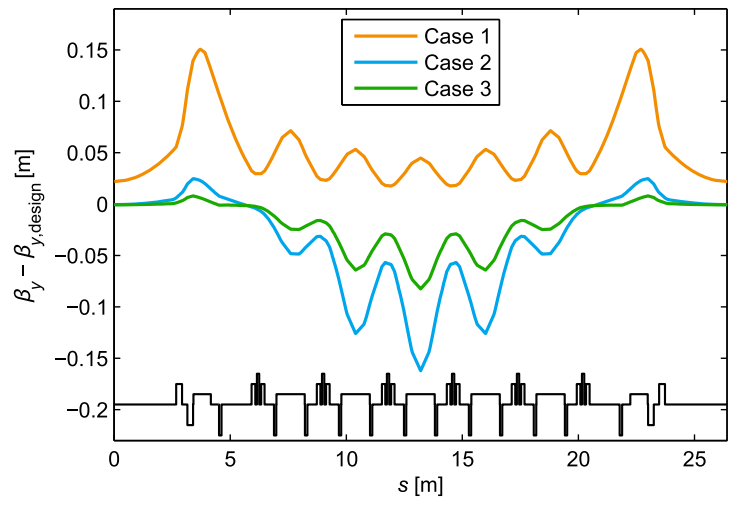

(b) Vertical beta functions

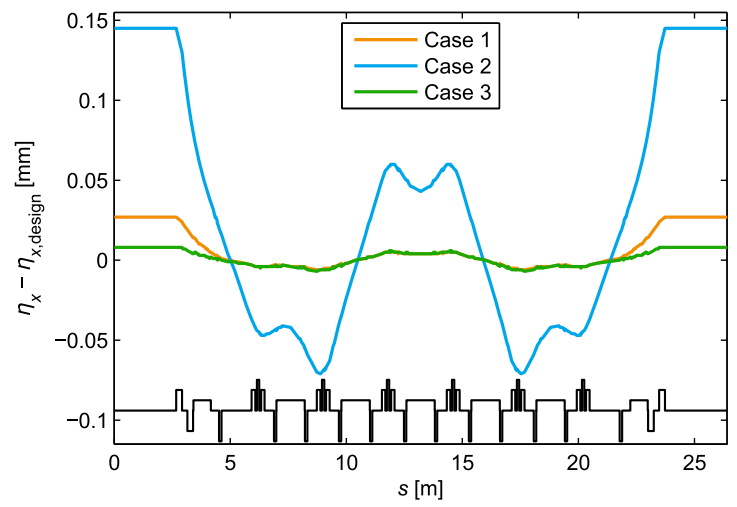

(c) Horizontal dispersion functions

FIG. 3. Difference between the design optics functions and the optics functions from SCVDB lattices. For all cases, the vertical emittance is $6.6 \mathrm{pm}$ rad in the ideal lattice and on average $7.9 \mathrm{pm}$ rad when including imperfections. 
is not closed but only suppressed in the long straights (Fig. 2). This case also shows considerable growth in the horizontal beta function compared to the design optics (Fig. 3(a)). The design of Case 2 makes use of two skew quadrupole families and is focused on minimizing the betatron coupling in the long straights, here an undesired side-effect of skew quadrupole excitation. Although the horizontal dispersion in the long straight appears comparably large [Fig. 3(c)], this example still shows advantages compared to Case 1. Case 3 shows a similar design as Case 2 but involves three skew quadrupole families, where the added family allows an efficient correction of the deficiencies of the previous cases. With the additional degree of freedom the defined target parameters can be approached more closely. However, further enforcing the target parameters does not appear to be of much practical benefit since the remaining discrepancies approach the level of deviations between ideal lattice and real lattice (i.e. lattices including imperfections, see Sec. III F).

\section{Scaling of SCVDBs}

The method introduced in Sec. II C provides SCVDB designs that can be easily scaled in vertical dispersion, generated vertical emittance and hence, Touschek lifetime as required. By adjusting the gradients proportionally in all skew quadrupoles, only the amplitude but not the general shape of the vertical dispersion bumps is changed [21], see Fig. 4. For adjusting the vertical emittance in practice the approximation $\mathcal{E}_{\mathrm{II}} \propto k^{2}$ [20] offers a simple way to scale without requiring a redesign via the sensitivity matrix approach. Since not only the vertical dispersion but also the deviations from the design optics scale with the skew quadrupole gradient it is useful to design SCVDBs with

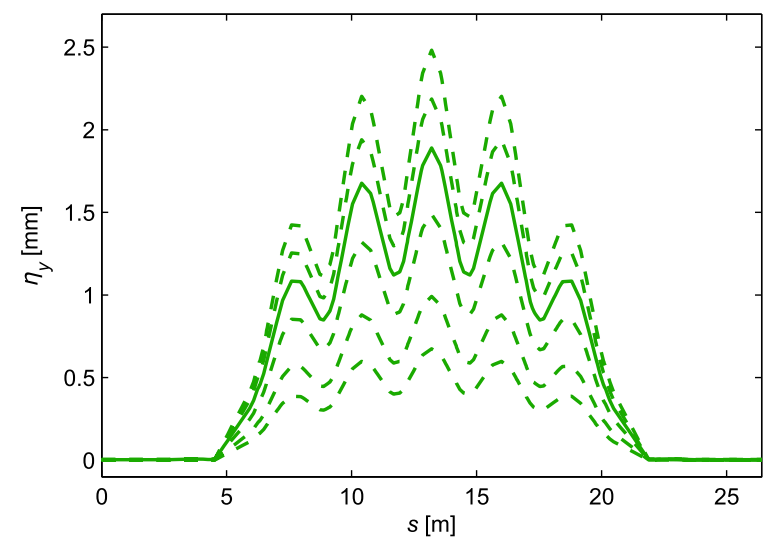

FIG. 4. SCVDB scaling in the ideal lattice of Case 3. Indicated with a solid line is the vertical dispersion that creates $6.6 \mathrm{pm}$ rad of vertical emittance in the ideal lattice and on average $7.8 \mathrm{pm}$ rad when including imperfections. The dashed lines show the vertical dispersions of scaled SCVDBs that create $\mathcal{E}_{\mathrm{II}}=0.9,1.8,4.1,8.9$ and $11.4 \mathrm{pm}$ rad in the ideal lattice, respectively.

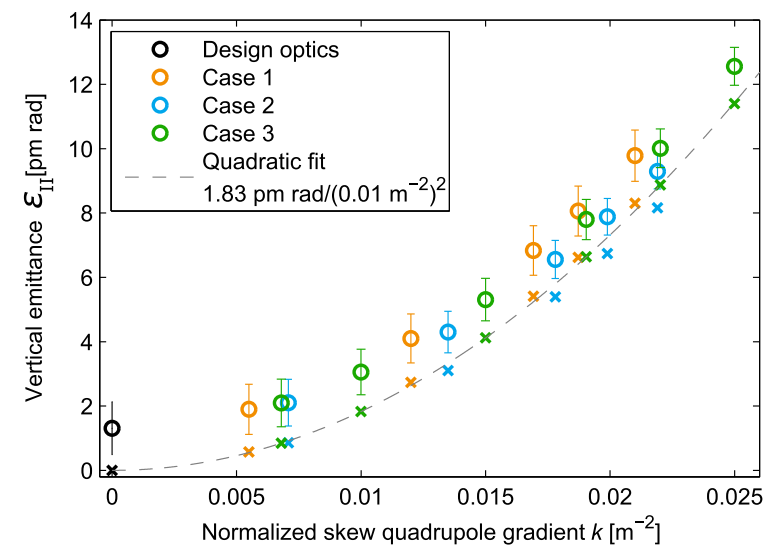

FIG. 5. Vertical emittance scales with skew quadrupole gradient squared. Ideal lattice (x) and error lattice (o) with standard deviations from 10 error seeds. Only the $k$ of the strongest skew quadrupole involved in each case is considered.

sufficient amplitude, followed by a down-scaling to the exact required vertical emittance. This ensures that the defined boundary constraints are not violated due to the scaling.

Scaling is demonstrated in Fig. 5, where the vertical emittance generated in Case 1,2, and 3 in the ideal lattice is shown as a function of the required normalized skew quadrupole gradient $k$. Since Cases 2 and 3 are based on more than one skew quadrupole family (see Table I for details), the strongest skew quadrupole is considered here. The quadratic fit for Case 3 gives $1.83 \mathrm{pm} \mathrm{rad}$ vertical emittance per $\left(0.01 \mathrm{~m}^{-2}\right)^{2}$ skew quadrupole gradient.

\section{Skew quadrupole gradients}

Skew quadrupole gradients are constrained by the available gradients in the real machine (magnet coils, power supplies), but also by the design goal of minimum perturbations of the design optics, especially avoiding nonlinear effects. Therefore, one design criterion is minimizing required skew quadrupole gradient while enforcing target parameters to sufficient degree. In all presented cases the normalized skew quadrupole gradient required to achieve $8 \mathrm{pm}$ rad vertical emittance (including lattice

TABLE I. Normalized skew quadrupole gradient $k$ in $\mathrm{m}^{-2}$, generated by auxiliary coils on the magnets used for the presented SCVDBs. The effective length of each magnet is $0.1 \mathrm{~m}$. The vertical emittance $\mathcal{E}_{\text {II }}$ given here is created in the ideal lattice (i.e. without magnet errors or misalignments).

\begin{tabular}{lcccc}
\hline \hline & OXX & SDend & SFm & $\mathcal{E}_{\text {II }}[\mathrm{pm} \mathrm{rad}]$ \\
\hline Case 1 & 0 & 0.0187 & 0 & 6.62 \\
Case 2 & 0.0198 & 0.0138 & 0 & 6.74 \\
Case 3 & 0.0193 & 0.0114 & -0.0017 & 6.64 \\
\hline \hline
\end{tabular}


imperfections) is less than $0.02 \mathrm{~m}^{-2}$ i.e. less than $20 \%$ of the maximum available gradient in these types of magnets. In comparison, the expected skew quadrupole gradient required to compensate for coupling effects from EPUs is approximately $0.03 \mathrm{~m}^{-2}$ [24].

\section{E. Impact on design optics and corrections}

Deviations of the beta functions from design values induce small changes in the phase advance over the achromats and, thus, shift the machine working point.

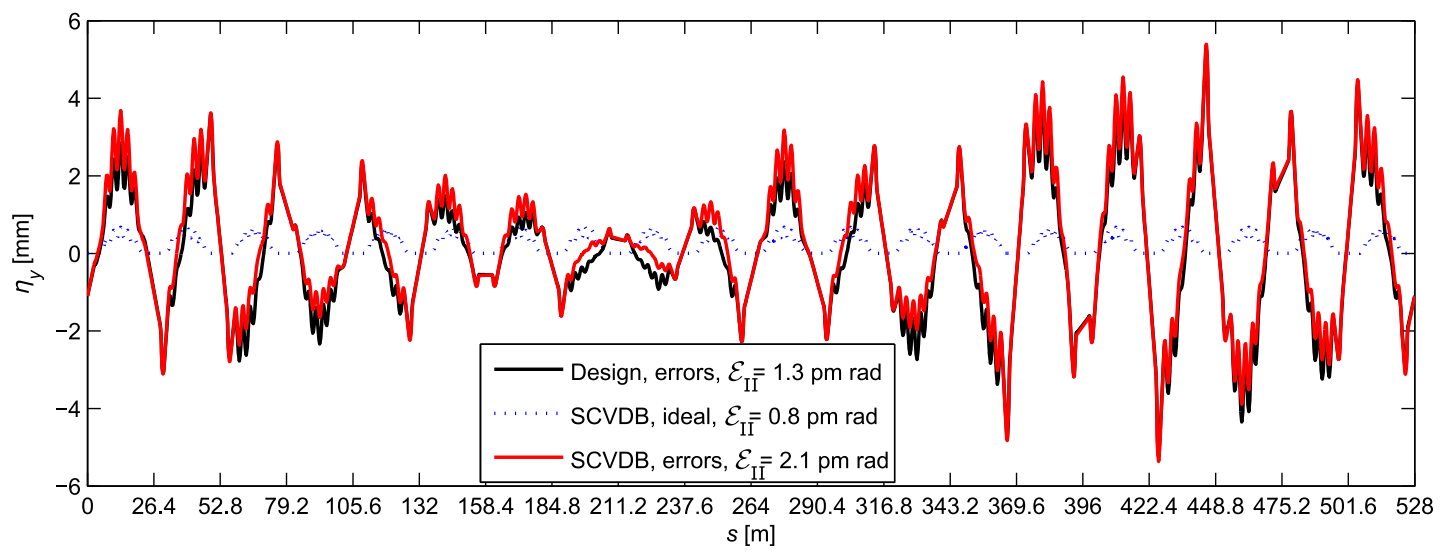

(a) Resulting vertical emittance, $\mathcal{E}_{\mathrm{II}}=2 \mathrm{pm}$ rad scaling.

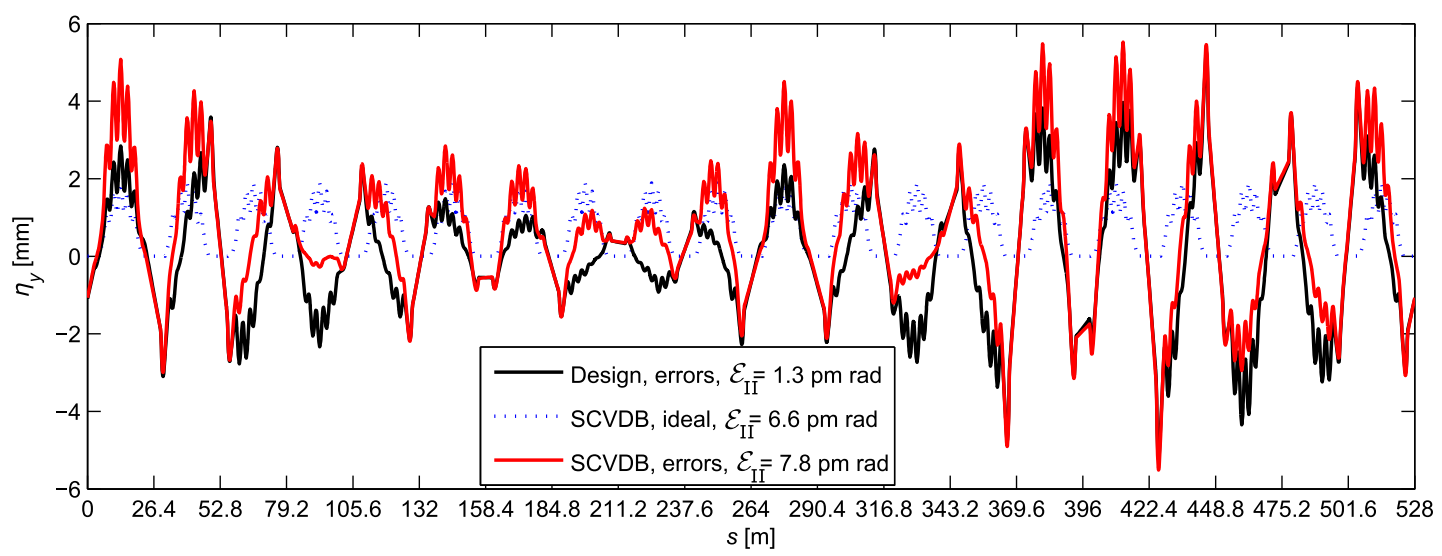

(b) Resulting vertical emittance, $\mathcal{E}_{\mathrm{II}}=8 \mathrm{pm}$ rad scaling.

FIG. 6. Vertical dispersion in the entire storage ring (20 achromats) for the design lattice and Case 3 lattice with identical error seed for two scalings. The ideal lattice SCVDB is shown for comparison.

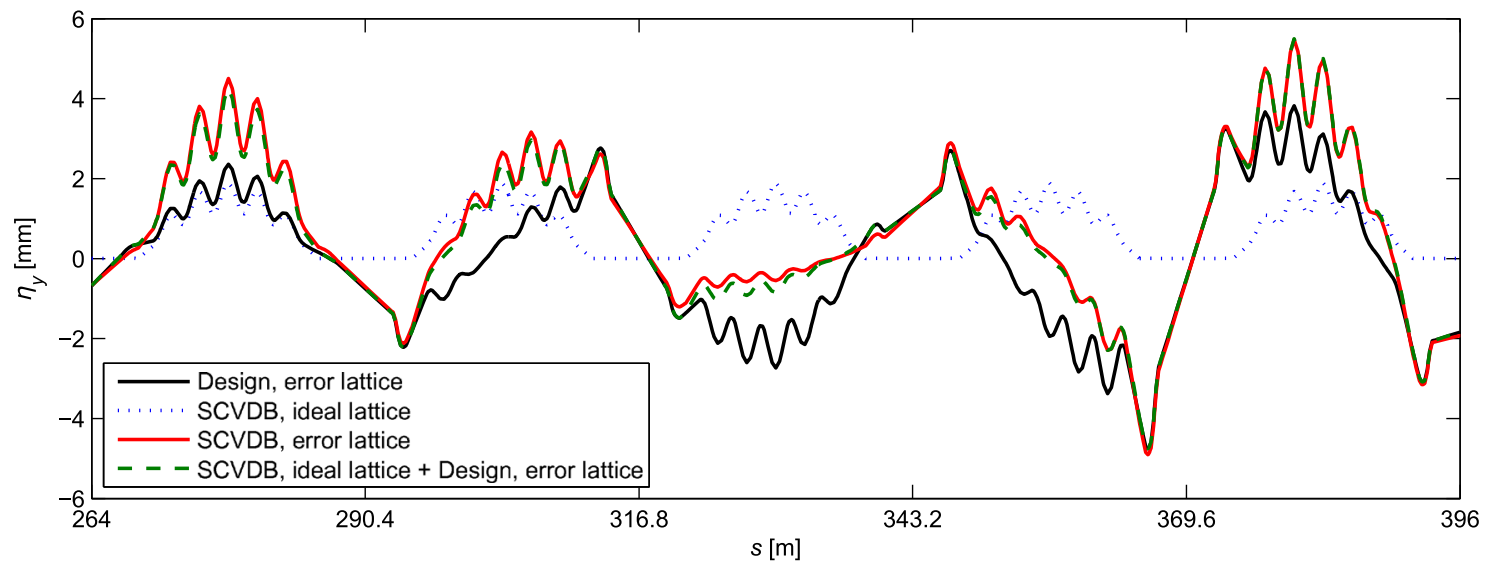

FIG. 7. Detail of vertical dispersion Case 3 in five achromats. Adding the vertical dispersion from the two independent sources, lattice imperfections and SCVDBs, linearly leads to a good estimate of the vertical dispersion under the influence of lattice errors. 
TABLE II. Impact of SCVDBs on beam sizes, vertical projected emittance in IDs (center of long straights), eigenemittances and on the emittance ratio $\kappa=\mathcal{E}_{\mathrm{II}} / \mathcal{E}_{\mathrm{I}}$. All results are averaged over 20 straight sections and 10 error seeds. The standard deviation is also displayed.

\begin{tabular}{lccrrr}
\hline \hline & $\sigma_{x}[\mu \mathrm{m}]$ & $\sigma_{y}[\mu \mathrm{m}]$ & $\epsilon_{y}[\mathrm{pm} \mathrm{rad}]$ & $\mathcal{E}_{\mathrm{I}}[\mathrm{pm} \mathrm{rad}]$ & $\mathcal{E}_{\text {II }}[\mathrm{pm} \mathrm{rad}]$ \\
\hline Design & $54.35 \pm 0.37$ & $1.89 \pm 0.14$ & $1.95 \pm 1.27$ & $328.3 \pm 0.4$ & $1.31 \pm 0.82$ \\
Case 1 & $55.29 \pm 0.43$ & $4.66 \pm 0.30$ & $12.35 \pm 1.05$ & $330.2 \pm 0.4$ & $8.06 \pm 0.78$ \\
Case 2 & $54.42 \pm 0.44$ & $4.13 \pm 0.09$ & $8.96 \pm 0.88$ & $327.4 \pm 0.3$ & $7.88 \pm 0.57$ \\
Case 3 & $54.35 \pm 0.40$ & $4.09 \pm 0.08$ & $8.53 \pm 0.97$ & $327.8 \pm 0.3$ & $7.80 \pm 0.63$ \\
\hline \hline
\end{tabular}

This is easily corrected by a slight global tuning of the main quadrupoles in the storage ring. The required changes in quadrupole gradient are less than $0.1 \%$. This adjustment of the quadrupole gradients can shift the linear chromaticity in both planes requiring a correction with at least two chromatic sextupole families. A change in sextupole gradient of less than $2 \%$ restores the linear chromaticities to the design values $\xi_{x, y}=+1.0$. These corrections are performed on the ideal lattice, before adding lattice imperfections to the simulation. Examples for deviations of higher order optics from design and their effect on the machine performance and beam lifetime are discussed in Sec. III I.

\section{F. Effect of lattice imperfections on SCVDBs}

Studies on error lattices are performed in order to estimate the performance of SCVDBs under realistic conditions. As in previous studies on the MAX IV $3 \mathrm{GeV}$ storage ring [23,25], the generated error lattices include the effects of misalignments of magnets, magnetic field errors and multipole errors. For each error seed orbit correction is applied, however, no minimization of betatron coupling or spurious vertical dispersion is attempted at this point, since the vertical emittance of the error lattices is $\approx 1.3 \mathrm{pm}$ rad and beam tilts from betatron coupling in the long straights are sufficiently small for user operation. The same 10 error seeds are applied to both the design lattice and to the three SCVDB cases. The increase in average vertical emittance due to errors varies between $1.1 \mathrm{pm}$ rad

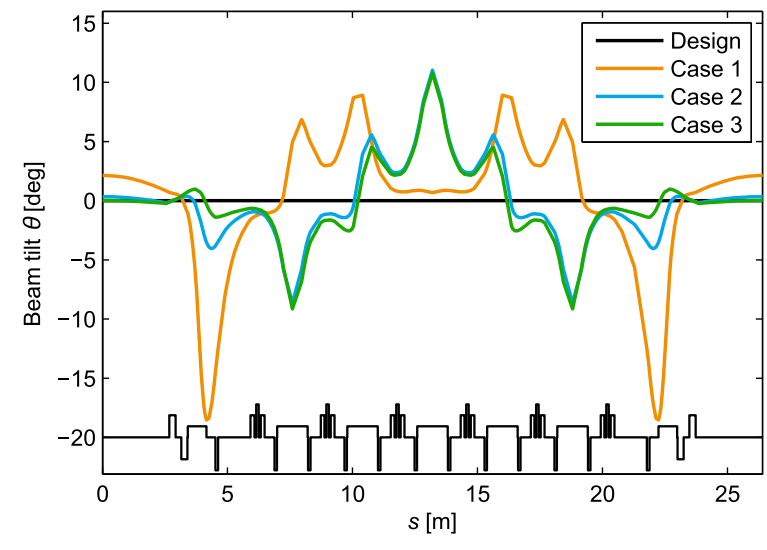

FIG. 8. Beam tilt angle $\Theta$ in the ideal lattice. and $1.4 \mathrm{pm} \mathrm{rad}$, depending on the individual SCVDB case and scaling.

The effect of errors on the Case 3 vertical dispersion function is shown in Fig. 6(a) and (b) for one error seed as an example. Note that orbit correction is applied but spurious vertical dispersion and betatron coupling are not minimized. For a desired vertical emittance of $2 \mathrm{pm}$ rad the necessary emittance increase by the SCVDB amounts to only half of the vertical emittance generated by errors. Therefore, the ideal vertical dispersion from the SCVDB is small compared to the vertical dispersion caused by errors in the design lattice [see Fig. 6(a)]. As expected, the vertical dispersion of the SCVDB lattices with errors does not deviate much from the vertical dispersion of the design lattice. For $8 \mathrm{pm}$ rad desired vertical emittance the SCVDBs are the dominant source of vertical emittance, since they create approximately $6.6 \mathrm{pm}$ rad. Figure 6(b) illustrates the working principle of the SCVDB lattice as a shift of the design vertical dispersion caused by random errors toward positive values. A relatively small increase in vertical dispersion is sufficient to achieve the targeted vertical emittance.

It is an interesting observation that the vertical dispersion of the SCVDB lattice without imperfections added linearly to the vertical dispersion in the design lattice generated randomly by imperfections, provides a good approximation $^{3}$ of the total vertical dispersion of the SCVDB lattices including errors. This behavior is shown in Fig. 7.

\section{G. Beam sizes, beam tilt and projected emittance}

Apart from the expected growth of the vertical beam size, following the approximate relation $\sigma_{y} \propto \sqrt{\mathcal{E}_{\text {II }}}$, only Case 1 shows an increased vertical beam size in the center of the long straight (Table II). The Case 1 lattice shows the largest deviations from design in terms of beta function [see Fig. 3(a) and (b)]. Furthermore, the betatron coupling created in the arc is not closed and a comparably large systematic beam tilt angle [18] is introduced in the long straight (see Fig. 8). In all cases the beam sizes are dominated by the betatronic contribution while the dispersive contributions can be neglected. This is in

\footnotetext{
${ }^{3}$ The error lattice contains among others rolled sextupoles as well as misaligned and rolled octupoles as nonlinear sources of dispersion, leading to deviations from a strictly linear behavior.
} 


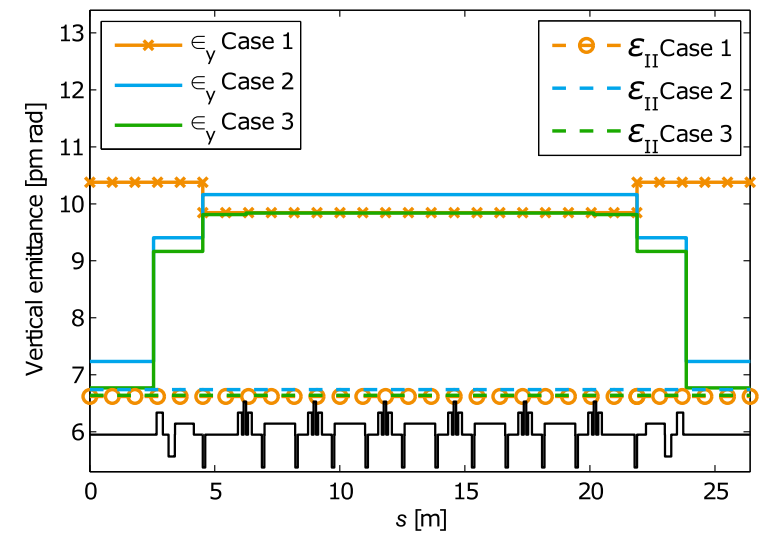

FIG. 9. Vertical projected emittance $\epsilon_{y}$ (solid lines) and vertical eigenemittance $\mathcal{E}_{\text {II }}$ (dashed lines) in the ideal lattice for the three cases. (Markers are displayed for Case 1 since traces partially overlap.)

accordance with the original design paradigm where maximum tolerable $\eta_{x, y}$ contributions were specified for the long straights (see Sec. III A).

Both electron beam size and divergence define the phase space surface occupied by the beam in the laboratory frame and form the projected emittance $\epsilon[19,26,27]$ as a relevant source parameter in terms of matching electron beam optics to the intrinsic radiation from IDs [5]. Unlike the constant eigenemittance $\mathcal{E}$, the projected emittance $\epsilon$ varies along the storage ring and is equal to the eigenemittance only in the absence of coupling. Both emittances in the vertical plane, $\epsilon_{y}$ and $\mathcal{E}_{\mathrm{II}}$, are shown in Fig. 9 for the three cases and excluding lattice imperfections. Only in Case 2 and Case 3 does $\epsilon_{y}$ approach $\mathcal{E}_{\text {II }}$ in the long straight sections, demonstrating the effective local betatron coupling suppression of these SCVDBs. Case 1 on the other hand shows an increase of vertical projected emittance in the long straight sections, amounting to $\epsilon_{y}=10.4 \mathrm{pm} \mathrm{rad}$ in the ideal lattice, and is therefore not compatible with the targeted diffraction limited operation with 1 A SR.

A slight improvement of Case 1 is, however, possible without much extra effort by changing the polarity of the skew quadrupoles in every other achromat. The result is a zero crossing (instead of a constant deviation from zero, see Fig. 2) of the vertical dispersion and a reduction of the beam tilt angle in the long straights (Fig. 8). Nevertheless, since the vertical dispersion and betatron coupling are not properly closed, the vertical projected emittance remains substantially higher than the vertical eigenemittance $\left(\epsilon_{y}=9.5 \mathrm{pm} \mathrm{rad}, \mathcal{E}_{\mathrm{II}}=6.6 \mathrm{pm} \mathrm{rad}\right)$.

When including the effects of lattice imperfections the vertical projected emittance of the design optics becomes $1.95 \mathrm{pm}$ rad averaged over 20 long straight sections and 10 error seeds (Fig. 10). This is an increase of $0.64 \mathrm{pm} \mathrm{rad}$ compared to the vertical eigenemittance. In the SCVDBs Case 1,2, and 3 the vertical projected emittance in the long straight sections exceeds the vertical eigenemittance by

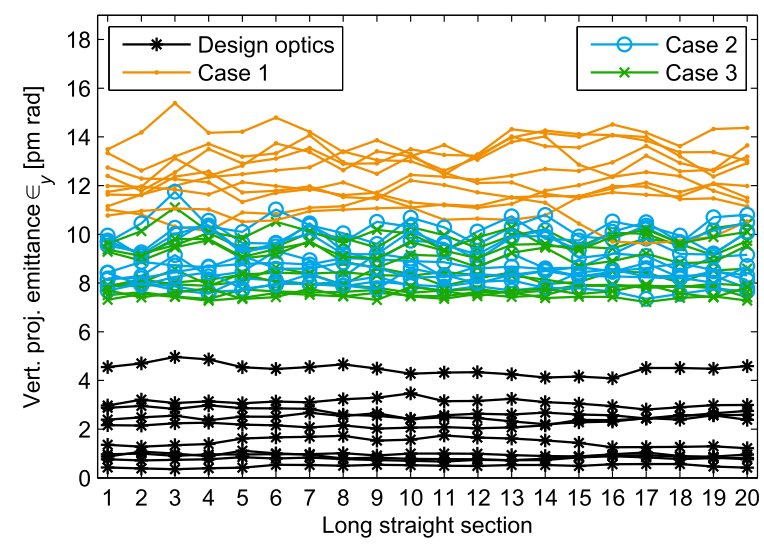

FIG. 10. Vertical projected emittance $\epsilon_{y}$ in the long straight sections (ID locations) including lattice imperfections (10 error seeds) for the design optics and the three SCVDB cases.

$4.29,1.08$, and $0.73 \mathrm{pm} \mathrm{rad}$, respectively, as shown in Table II.

\section{H. Touschek lifetime results from tracking}

Touschek lifetime was calculated from local lattice momentum acceptance derived from tracking in 6D around the entire storage ring with Tracy-3. The Touschek lifetime calculated here, however, does not take into account bunch elongation from Landau cavities which increases the Touschek lifetime by roughly a factor five [4] independently of the vertical emittance adjustments detailed here. Lattice errors are included in the simulations and the vertical aperture is globally limited to $\pm 10 \mathrm{~mm}$, corresponding to the vertical acceptance of the vacuum system without in-vacuum undulators or narrow-gap chambers. The tracking is performed over a full synchrotron period which in the MAX IV $3 \mathrm{GeV}$ storage ring (without Landau cavities) is $\tau_{s}=880 \mu \mathrm{s}$ (500 turns). Results are displayed in Table III.

Under the approximation $\tau \propto \sqrt{\mathcal{E}_{\text {II }}}$ (see Sec. II A) the Touschek lifetime gain achieved with SCVDBs compared to the design lattice can be quantified with a Figure of Merit (FOM) as follows:

$$
\mathrm{FOM}_{i}=\frac{\tau_{i}}{\hat{\tau}_{i}} \cdot \sqrt{\frac{\hat{\mathcal{E}}_{\mathrm{II}, i}}{\mathcal{E}_{\mathrm{II}, i}}}
$$

TABLE III. Tracking results for Touschek lifetime along with FOM for each case. Mean value and standard deviation are given for 10 error seeds.

\begin{tabular}{lcrc}
\hline \hline & $\mathcal{E}_{\text {II }}[\mathrm{pm} \mathrm{rad}]$ & \multicolumn{1}{c}{$\tau[\mathrm{h}]$} & FOM \\
\hline Design & $1.31 \pm 0.82$ & $5.06 \pm 1.64$ & 1 \\
Case 1 & $8.06 \pm 0.78$ & $11.23 \pm 0.36$ & $0.854 \pm 0.017$ \\
Case 2 & $7.88 \pm 0.57$ & $12.12 \pm 0.58$ & $0.931 \pm 0.033$ \\
Case 3 & $7.80 \pm 0.63$ & $12.55 \pm 0.51$ & $0.969 \pm 0.019$ \\
\hline \hline
\end{tabular}




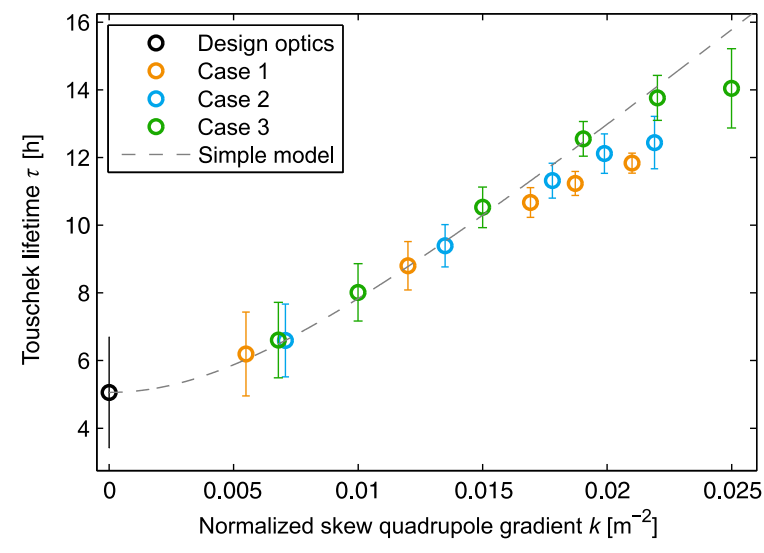

FIG. 11. Touschek lifetime as a function of skew quadrupole gradient. Tracking with errors, average and standard deviation for 10 seeds. Only the gradient of the strongest skew quadrupole used in each case is considered in this plot.

where $\hat{\tau}$ and $\hat{\mathcal{E}}_{\text {II }}$ relate to the design lattice, $\tau$ and $\mathcal{E}_{\text {II }}$ relate to the SCVDB lattice, and $i$ is an identifier for the error seed. Thus, a resulting FOM $<1$ indicates that the Touschek lifetime gain is lower than expected from the simple $\tau \propto$ $\sqrt{\mathcal{E}_{\text {II }}}$ relation. Possible reasons for this behavior are discussed in the following section. The FOM as an average over 10 error seeds is shown in Table III for the three SCVDB lattice designs. It is important to note that the FOM must be calculated for the individual error seed, as indicated in Eq. (9), in order to be a realistic measure of the performance of a SCVDB lattice under different conditions of lattice imperfections. Scaling averaged lifetimes with averaged vertical emittances will not lead to the same result.

A rough estimate of the general performance of the presented cases in terms of Touschek lifetime $\tau$ as a function of the normalized skew quadrupole gradient $k$, based on the relation $\tau \propto \sqrt{\mathcal{E}_{\text {II }}}$, and including the effect of lattice imperfections is given by

$$
\tau=a \sqrt{b \cdot k^{2}+\left\langle\hat{\mathcal{E}}_{\mathrm{II}}\right\rangle},
$$

where $\left\langle\hat{\mathcal{E}}_{\text {II }}\right\rangle=1.31 \mathrm{pm}$ rad is the vertical emittance of the design lattice, including lattice imperfections and averaged over 10 error seeds. The fitted parameters are $a=4.42$ hours Touschek lifetime per $\sqrt{\mathrm{pm} \mathrm{rad}}$ of vertical emittance and $b$, the $1.83 \mathrm{pm}$ rad vertical emittance per $\left(0.01 \mathrm{~m}^{-2}\right)^{2}$ skew quadrupole gradient, mentioned in Sec. IIIC. The curve based on this simple model, representing a $\mathrm{FOM}=1$, is shown, together with the tracking results for Touschek lifetime, in Fig. 11.

\section{Discrepancies in lifetime gain}

Generally, the FOM is higher for lower skew quadrupole excitation and thus SCVDBs closer to the design lattice.

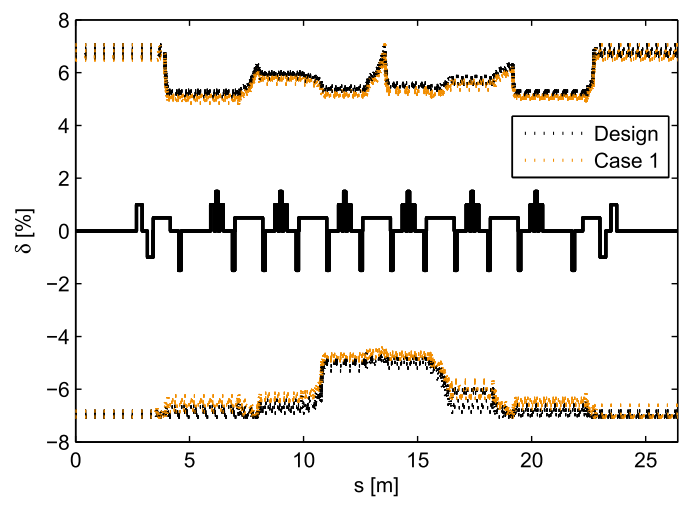

(a) Case 1

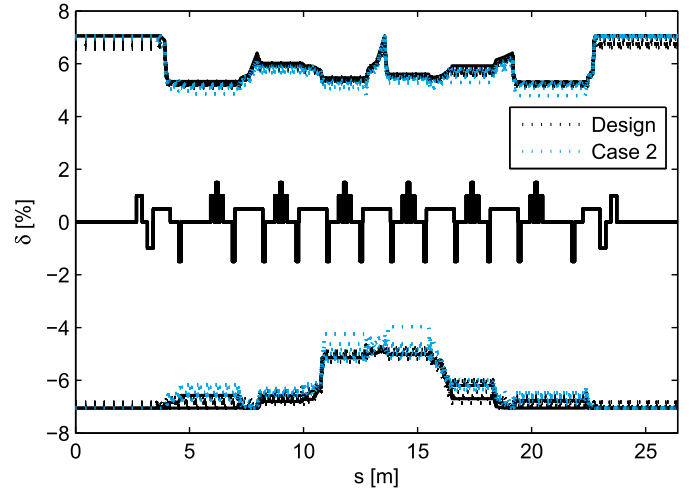

(b) Case 2

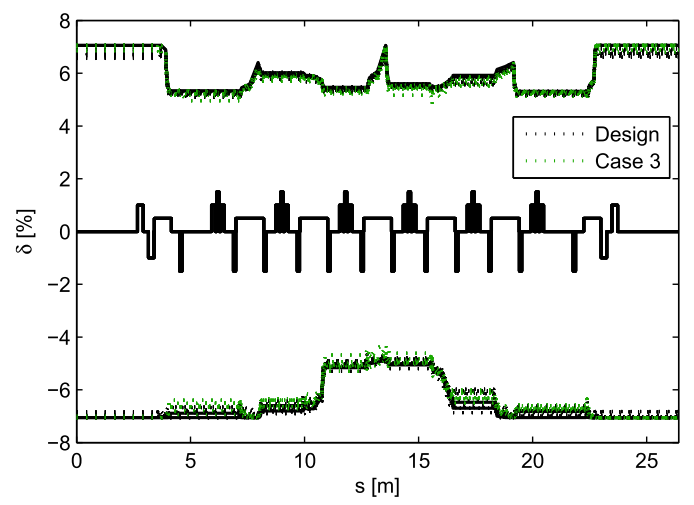

(c) Case 3

FIG. 12. Momentum aperture from 10 error seeds for the 3 cases, compared to design lattice (black) in the first achromat. The SCVDB lattices are scaled to approximately $\mathcal{E}_{\mathrm{II}}=8 \mathrm{pm} \mathrm{rad}$ while the design lattice with errors is on average at $1.3 \mathrm{pm}$ rad.

This is illustrated in Fig. 11, where the deviation from the theoretical, ideal behavior becomes larger with increasing skew quadrupole gradient. Also, the differences between the SCVDB lattices become apparent, since designs that fulfill the target parameters to higher degree show less deviation from the theoretical assumption of $\mathrm{FOM}=1{ }^{4}$

\footnotetext{
${ }^{4}$ Situations with FOM $<1$ can occur if the dispersive contribution to the vertical beam size is not negligible. However, this is in contradiction to the initial assumptions.
} 


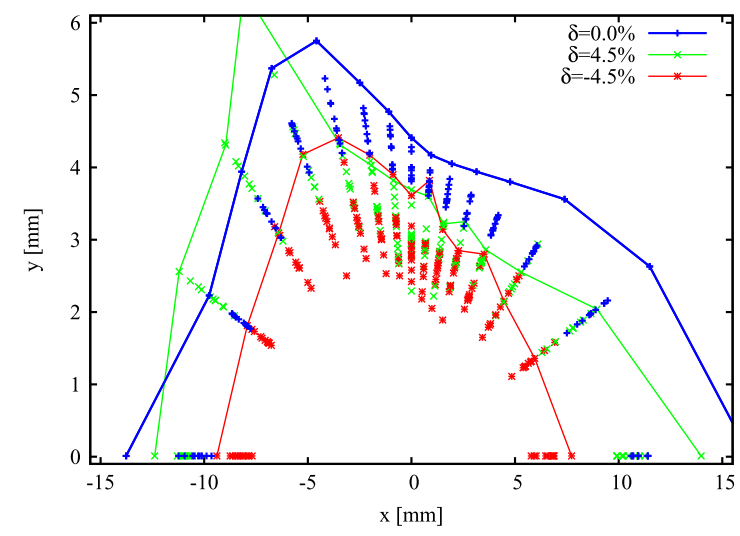

(a) Design

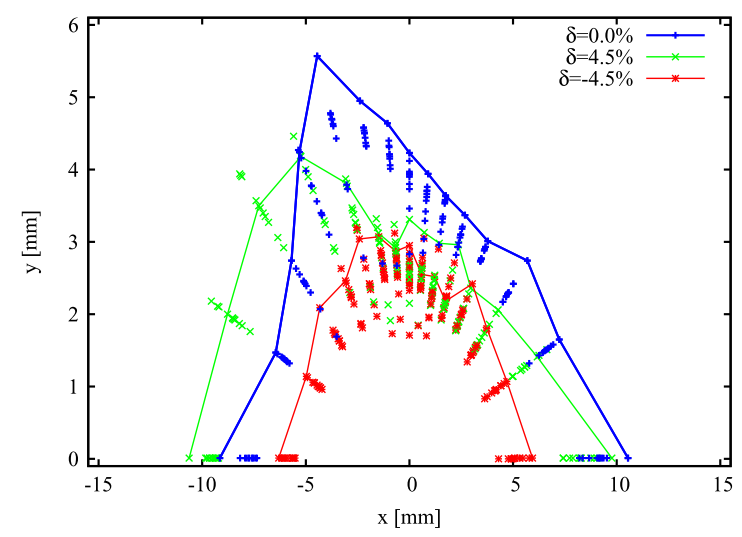

(c) Case 2

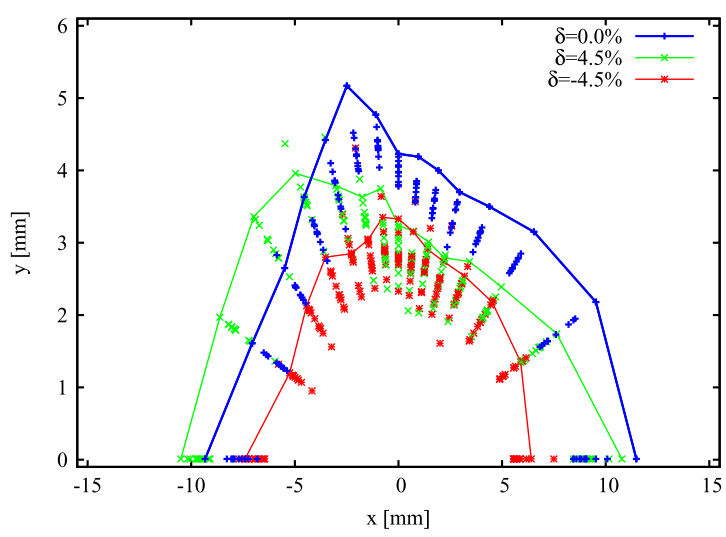

(b) Case 1

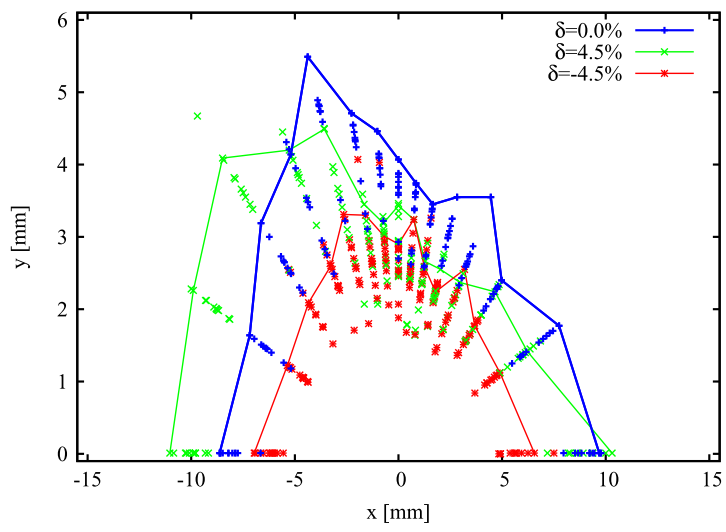

(d) Case 3

FIG. 13. Dynamic aperture for the ideal lattice and 10 error seeds. (a) shows the design lattice and (b)-(d) show the three SCVDB cases. The DA was calculated at the center of the first long straight section, close to the injection point.

To study the effects in detail that lead to beam loss and therefore reduced Touschek lifetime we investigated momentum aperture (MA), dynamic aperture, amplitude dependent tune shift (ADTS) and nonlinear chromaticity for all 10 error seeds. The tendency of a larger MA with increasing number of involved skew quadrupoles supports the design paradigm of minimizing deviations from design optics in order to maximize SCVDB performance. The MAs shown for the three cases and the design lattice can be found in Fig. 12. Although the differences are small, Case 3 shows the least restrictions in MA and is closest to the MA of the design lattice. This agrees with the fact that the FOM approaches 1 even at vertical emittances of $8 \mathrm{pm}$ rad for this SCVDB design.

The DA was simulated in the center of the first long straight section $(s=0)$, not far from the injection point [11]. For the MAX IV $3 \mathrm{GeV}$ ring injection scheme (on-energy, off-axis) the on-energy horizontal DA is the relevant parameter in terms of injection efficiency and ideally needs to be maintained at or beyond $7 \mathrm{~mm}$. Figure 13 shows that with lattice errors included the DA stays within tolerable limits.
Generally, the small deviations from design in the linear optics, followed by a correction of betatron tunes and linear chromaticity keep the ADTS within acceptable limits in the three presented SCVDB cases. Performance limitations in terms of Touschek lifetime that originate in restricted local MA may, however, be explained by increased ADTSs. For example, Case 1 shows large negative horizontal tune shift with vertical amplitude [Fig. 14(b)] compared to the design optics and the Case 3 optics [Fig. 14(a) and (c)]. Also, for Case 3 only slight changes of the chromatic tune shifts compared to the design lattice can be recognized (see Fig. 15).

In principle the ADTS as well as the quadratic chromaticity can be corrected after the implementation of a dispersion bump, with expected positive effects on the beam lifetime and the injection efficiency. For such corrections the MAX IV $3 \mathrm{GeV}$ storage ring is equipped with three families of octupole magnets (where corrections of ADTS and quadratic chromaticity are both first order effects) and five families of sextupole magnets in various locations. During the original lattice design the ADTS and quadratic chromaticity were optimized with these magnets 


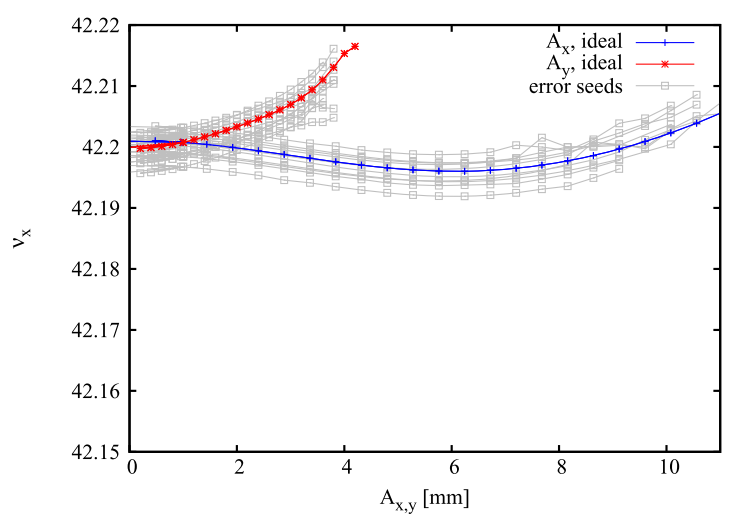

(a) Design

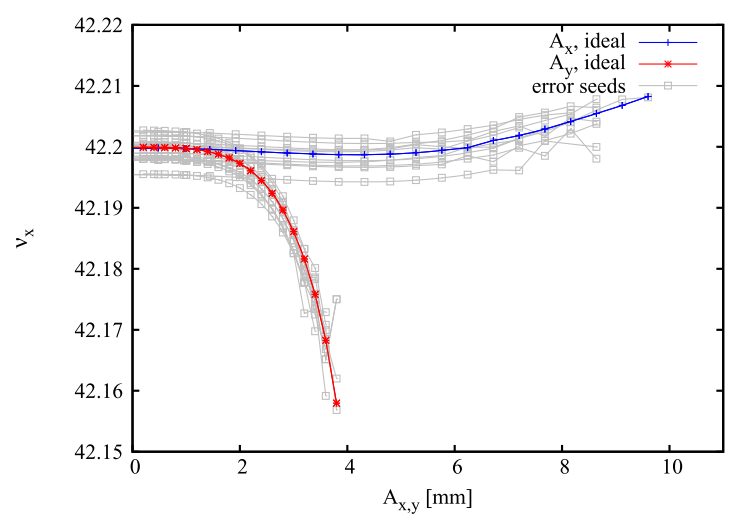

(b) Case 1

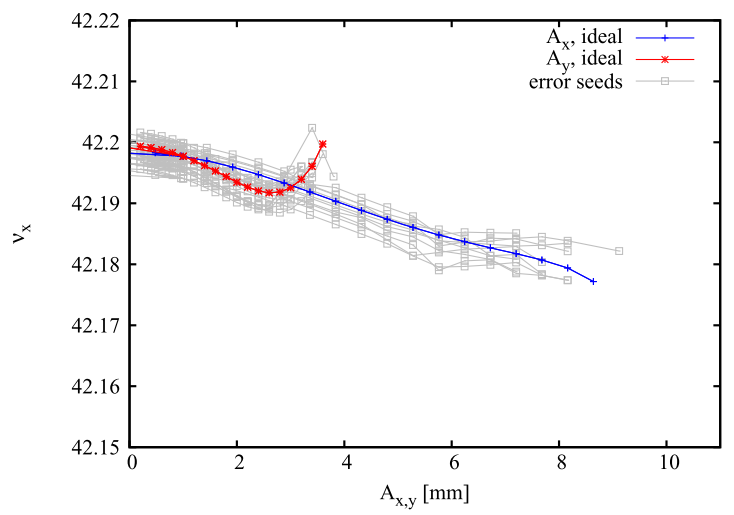

(c) Case 3

FIG. 14. Amplitude dependent tune shifts [horizontal tune $\left(\nu_{x}\right)$ ] for the design lattice and Case 1 and 3 SCVDB lattices, adjusted for $\mathcal{E}_{\text {II }}=6.6 \mathrm{pm} \mathrm{rad}$ without errors. Ideal lattice in blue (horizontal) and red (vertical). Ten error seeds in gray.

$[10,28]$. In this study, however, we focus on a SCVDB implementation with minimum impact on the design lattice. Corrections of ADTS and quadratic chromaticity with octupoles, combined with detailed frequency map analysis remain, therefore, as an option for further performance improvement, but are, as shown here, not inevitable for good SCVDB performance.

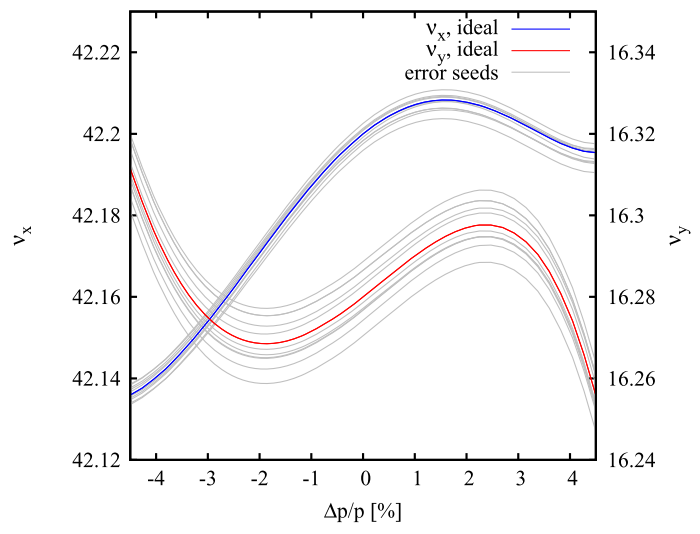

(a) Design

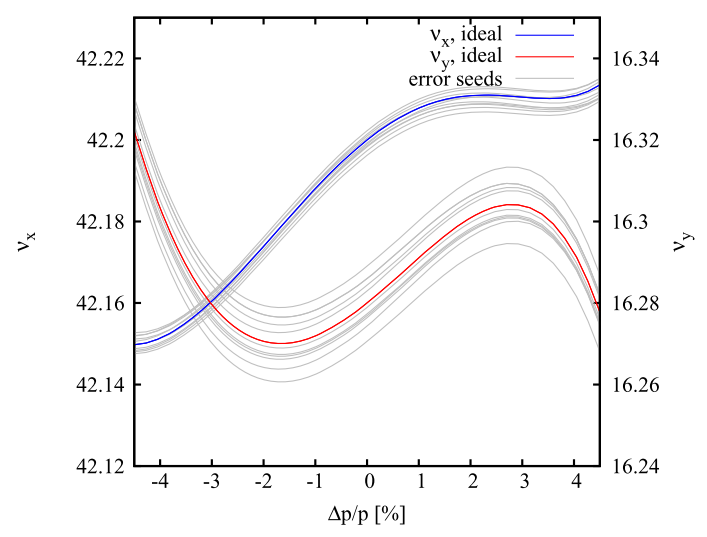

(b) Case 3

FIG. 15. Chromatic tune shifts for the design lattice and the Case 3 SCVDB lattice, adjusted for $\mathcal{E}_{\text {II }}=6.6 \mathrm{pm} \mathrm{rad}$ without errors. Ideal lattice in blue (horizontal) and red (vertical). Ten error seeds in gray.

\section{SUMMARY}

We have presented a systematic way of increasing the Touschek lifetime by employing successive closed vertical dispersion bumps that generate vertical emittance in a storage ring in a controlled fashion. By increasing the vertical emittance from 1.3 to $8 \mathrm{pm}$ rad we can increase the Touschek lifetime by a factor 2 to 2.5 with normalized skew quadrupole gradients of $0.02 \mathrm{~m}^{-2}$ in the MAX IV $3 \mathrm{GeV}$ storage ring. For a high-brightness operation mode [5] cases with a lower skew quadrupole excitation, leading to a vertical emittance of $2 \mathrm{pm}$ rad and a Touschek lifetime increase by $30 \%$, have been studied as well.

The systematic SVD-based approach allows a thoughtful, reasonable trade-off between the targeted lifetime increase, required effort and possible risks in terms of deviations from design optics. By keeping deviations from design optics low the dynamic aperture of the design lattice is preserved and injection efficiency remains high.

Employing just two independently excited skew quadrupole families, deviations from design optics of the MAX IV 
$3 \mathrm{GeV}$ storage ring can be reduced to a level that is comparable to deviations caused by lattice imperfections. Since vertical dispersion and betatron coupling are restricted to the arcs the source properties for IDs are not significantly altered except for the growth in vertical beam size due to the desired vertical emittance increase. By adding a third skew quadrupole family residual betatron coupling and vertical dispersion in the ID source points can be further reduced, however, considering the benefit during actual user operation the additional effort might not be justified.

Finally it remains to be emphasized that the presented approach to SCVDBs, starting from an ideal design lattice, is applicable to any ULE storage ring as long as a sufficient number of skew quadrupoles is available.

\section{ACKNOWLEDGMENTS}

One of the authors (J. B.) would like to thank Teresia Olsson for her helpful advice on the Tracy-3 code.

[1] C. Bernardini, G. F. Corazza, G. Di Giugno, G. Ghigo, J. Haissinski, P. Marin, R. Querzoli, and B. Touschek, Lifetime and Beam Size in a Storage Ring, Phys. Rev. Lett. 10, 407 (1963).

[2] H. Bruck, Accélérateurs circulaires de particules (Presses Universitaires de France, Paris, 1966).

[3] A. Streun, Report No. SLS Note 18/97, https://ados.web .psi.ch/slsnotes/sls1897a.pdf.

[4] S. C. Leemann, Interplay of Touschek scattering, intrabeam scattering, and rf cavities in ultralow-emittance storage rings, Phys. Rev. ST Accel. Beams 17, 050705 (2014).

[5] S. C. Leemann and M. Eriksson, Coupling and brightness considerations for the MAX IV $3 \mathrm{GeV}$ storage ring, in Proceedings of the 25th Particle Accelerator Conference, PAC, Pasadena, CA, 2013 (IEEE, New York, 2013), MOPHO05, p. 243.

[6] C. Steier, E. Forest, L. Nadolski, H. Nishimura, D. Robin, W. Wan, Y. Wu, and A. Zholents, Accelerator physics challenges of the fs-slicing upgrade at the ALS, in Proceedings of the 20th Particle Accelerator Conference, PAC, Portland, OR, 2003 (IEEE, New York, 2003), WOAB006, p. 397.

[7] C. Steier, D. Robin, A. Wolski, G. Portmann, and J. Safranek, Coupling correction and beam dynamics at ultralow vertical emittance in the ALS, in Proceedings of the 20th Particle Accelerator Conference, PAC, Portland, OR, 2003 (IEEE, New York, 2003), RPPG018, p. 3213.

[8] C. Montag, J. Bengtsson, and B. Nash, Touschek lifetime calculations and simulations for NSLS-II, in Proceedings of the 22nd Particle Accelerator Conference, PAC, Albuquerque, NM, 2007 (IEEE, New York, 2007), FRPMS113, p. 4375.

[9] J. Bengtsson, Tracy-2 User's Manual (unpublished).
[10] S. C. Leemann, A. Andersson, M. Eriksson, L.-J. Lindgren, E. Wallén, J. Bengtsson, and A. Streun, Beam dynamics and expected performance of Sweden's new storage-ring light source: MAX IV, Phys. Rev. ST Accel. Beams 12, 120701 (2009).

[11] S. C. Leemann, Pulsed sextupole injection for Sweden's new light source MAX IV, Phys. Rev. ST Accel. Beams 15, 050705 (2012).

[12] L. Liu, N. Milas, A. H. C. Mukai, X. R. Resende, and F. H. de Sá, The Sirius project, J. Synchrotron Radiat. 21, 904 (2014).

[13] J. Biasci, J. Bouteille, N. Carmignani, J. Chavanne, D. Coulon, Y. Dabin, F. Ewald, L. Farvacque, L. Goirand, M. Hahn et al., A low-emittance lattice for the ESRF, Synchrotron Radiat. News 27, 8 (2014).

[14] J. Safranek, Experimental determination of storage ring optics using orbit response measurements, Nucl. Instrum. Methods Phys. Res., Sect. A 388, 27 (1997).

[15] G. Wang, L. Yu, Y. Li, T. Shaftan, and L. Yang, NSLS-II storage ring coupling measurement and correction, in Proceedings of IPAC, Richmond, USA, 2015 (IEEE, New York, 2015), TUPHA009, p. 1983.

[16] A. Wolski, Alternative approach to general coupled linear optics, Phys. Rev. ST Accel. Beams 9, 024001 (2006).

[17] A. Wolski, D. Rubin, D. Sagan, and J. Shanks, Lowemittance tuning of storage rings using normal mode beam position monitor calibration, Phys. Rev. ST Accel. Beams 14, 072804 (2011).

[18] A. W. Chao and M. J. Lee, Particle distribution parameters in an electron storage ring, J. Appl. Phys. 47, 4453 (1976).

[19] A. W. Chao, Evaluation of beam distribution parameters in an electron storage ring, J. Appl. Phys. 50, 595 (1979).

[20] G. Guignard, Reports No. CERN-ISR-BOM-79-30 and No. CERN-LEP-Note-154, 1979.

[21] J. Bengtsson and I. Pinayev, Report No. NSLS-II Tech Note 7, 2007.

[22] W. H. Press, S. A. Teukolsky, W. T. Vetterling, and B. P. Flannery, Numerical Recipes: The Art of Scientific Computing, 3rd ed. (Cambridge University Press, Cambridge, England, 2007).

[23] MAX IV Detailed Design Report, http://www.maxiv.lu.se/ publications/, 2010.

[24] S. C. Leemann and H. Tarawneh, Impact of Insertion Devices on the MAX IV Storage Rings, in Proceedings of IPAC, Richmond, USA, 2015 (IEEE, New York, 2015), TUPJE038, p. 1696.

[25] S. C. Leemann, MAX IV Internal Note 20121107, http:// www.maxiv.lu.se/publications/.

[26] K. Ohmi, K. Hirata, and K. Oide, From the beam-envelope matrix to synchrotron-radiation integrals, Phys. Rev. E 49, 751 (1994).

[27] A. Franchi, L. Farvacque, J. Chavanne, F. Ewald, B. Nash, K. Scheidt, and R. Tomás, Vertical emittance reduction and preservation in electron storage rings via resonance driving terms correction, Phys. Rev. ST Accel. Beams 14, 034002 (2011).

[28] S. C. Leemann and A. Streun, Perspectives for future light source lattices incorporating yet uncommon magnets, Phys. Rev. ST Accel. Beams 14, 030701 (2011). 